\title{
Preoperative diagnosis of obscure gastrointestinal bleeding due to a GIST of the jejunum: a case report
} Stavros Gourgiotis*1,4, Dimitrios Kotoulas 1,5, Stavros Aloizos ${ }^{2,6,7}$, Aikaterini Kolovou ${ }^{3,7}$, Nikolaos S Salemis ${ }^{1,8}$ and Ioannis Kantounakis ${ }^{3}$

Address: ${ }^{1}$ Second Surgical Department, 401 General Army Hospital of Athens, Greece, ${ }^{2}$ Intensive Care Unit, 401 General Arp 4 Hospital of 5 , hens, Greece, ${ }^{3}$ Radiology Department, 401 General Army Hospital of Athens, Greece, ${ }^{4} 41$ Zakinthinou Street, 15669, Papagov. Atı. Gree 2,537 Alexandroupoleos Street, 12135, Peristeri, Athens, Greece, ${ }^{6} 14$ Acheon Street, 15343, Ag. Paraskevi, Athens, Greece, 724 Mykino tr et, Dafni, Athens, Greece, 819 Taxiarhon Street, 19014 Kapandriti, Athens, Greece and ${ }^{9} 1$ Thalias Street, Alimos, Athens, Gree

Email: Stavros Gourgiotis* - drsgourgiotis@tiscali.co.uk; Dimitrios Kotoulas - dkotoulas@hotmail.com; Stavr/s A Aikaterini Kolovou - katerinankolovou@yahoo.gr; Nikolaos S Salemis - nikos_salemis@ hotmail.com; Ioannis Kantounakis - yannis.kandounakis@gmail.com

* Corresponding author

Published: 25 November 2009

Cases Journal 2009, 2:9088 doi:10.1186/1757-1626-2-9088

This article is available from: http://www.casesjournal.com/content/2/I/9088 (C) 2009 Gourgiotis et al; licensee BioMed Central Ltd. This is an Open Access article distributed under the terms of the Creative Co which permits unrestricted use, distribution, and reproduction in any medir

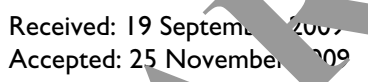

Accepted: 25 Novembe, 109

\begin{abstract}
Gastrointestinal stromal tumour ( $C$ Ts) are rare mesenchymal neoplasms affecting the digestive tract or nearby structures with the at men. We present a case of a 66-year-old female patient who presented with obscu e anemia due to gastrointestinal bleeding and underwent exploratory laparotomy during which large SIST of the small intestine was discovered. Examining the preoperative results of vid ondoscopy, computed tomography, and angiography and comparing them with nerative findings we discuss which of these investigations plays the most important role in the dite, cion and localization of GIST. A sort review of the literature is also conducted on se rale mesenchymal tumours.
\end{abstract}

\section{Introductirs}

Gastroint $c$ tinal stromal tumours (GISTs), which arise primarily h ge gull, are uncommon mesenchymal, mali or or the g2. Dir antinal tract. GISTs are the most common non epits al tumors of the digestive tract, accounting for only 1 , of all gastrointestinal malignancies $[1,2]$ and for $5.7 \%$ of all sarcomas [3]. These tumours are defined as specific, generally Kit (CD117)-positive and Kit or platelet-derived growth factor receptor alpha (PDGFRA) mutation-driven tumours [4].

This paper reports a case of obscure gastrointestinal bleeding due to a large GIST of the jejunum evaluates the role of preoperative investigations of this association and provides a short English literature review.

\section{Case Report}

A 66-year-old Caucasian female with no past medical history presented with a 3-month history of anemia. There was no associated fever, dyspeptic symptoms, nausea, weight loss, vomiting, or localized abdominal pain. Physical examination and blood biochemistry were within normal rates. Hematologic tests showed decrease of hematocrit (Ht: 19\%) and platelets (PLT) count of $76,000 / \mu \mathrm{L}$. The patient was HIV 1-2 negative. CEA and CA $19-9$ were in the normal range. 
Chest and abdominal X-rays, abdominal ultrasonography (US), upper gastrointestinal endoscopy, and colonoscopy were unremarkable. Abdominal computed tomography (CT) showed a well-delimited lobulated large mass measuring $10 \times 9 \times 5 \mathrm{~cm}$ in the jejunum with malignant behavior. Video capsule endoscopy (VCE) revealed an extensive venous plexus giving the possible diagnosis of angiodysplasia of small intestine. Angiography demonstrated voluminous disorder with abnormal arterial structures and areas of stagnation of the contrast medium (Figures 1,2). Due to the extension of the disorder, the embolization was abandoned.

The patient underwent exploratory laparotomy. During surgery, a well-circumscribed lesion, measuring $10.5 \times 9.5$ $\times 6 \mathrm{~cm}$ was identified at the jejunum (Figure 3 ). A complete resection of the mass and a side to side anastomosis of the small bowel were performed. The patient had an uneventful postoperative course and was discharged on the sixth postoperative day.

The histopathological examination of the resected lesion revealed a mesenchymal tumour categorized as GIST tumour. The stromal tumour demonstrated whirling sheets of spindle cells with moderate level of phenomorphism and mitotic activity (6-7 mitoses/50 HPF) (H\&E stain). No necrosis was observed. Immunohistocherical staining for CD117, $\alpha$-smooth-muscle actin (SMA), a is

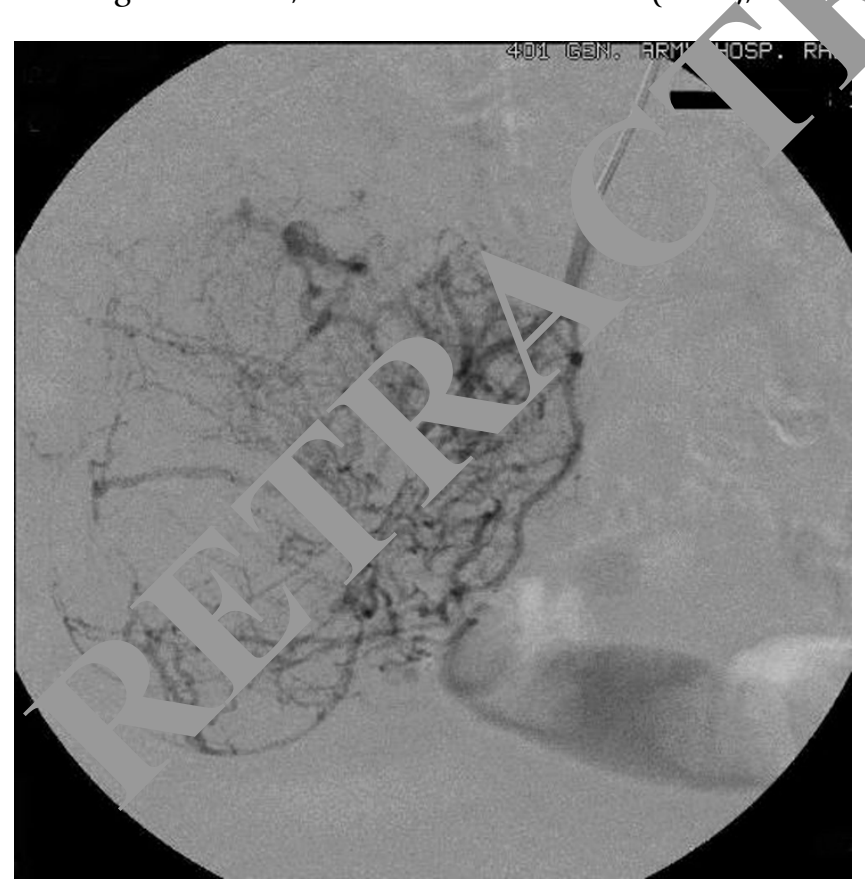

Figure I

The superselective catheterization of an ileal artery shows voluminous disorder with abnormal arterial structures and areas of stagnation of the contrast medium.

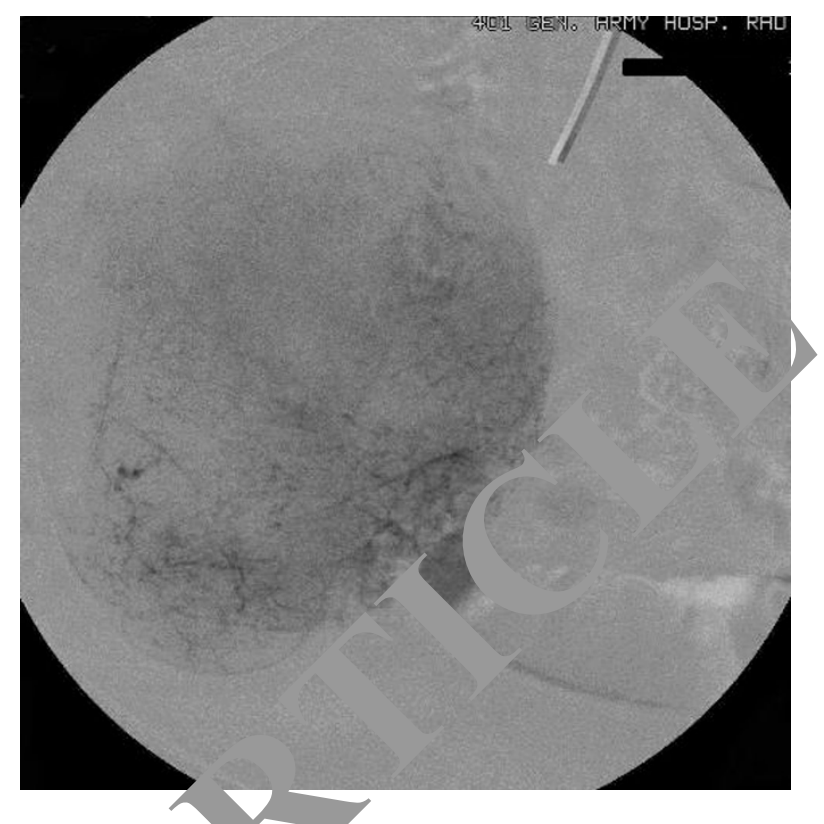

Figure 2

The par -hymal, hase of the superselective catheterization 0 . same ileal artery shows the full extent of ine disorder.

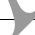

$00 \mathrm{p}$ otein was positive, whereas staining for desmin and - 24 was negative. The labeling index for MIB-1, determined by counting positively stained nuclei, was about $5 \%$.

\section{Discussion}

The term GIST was introduced by Mazur and Clark in 1983 in order to indicate a distinct heterogeneous group of mesenchymal neoplasms of spindle or epithelioid cells

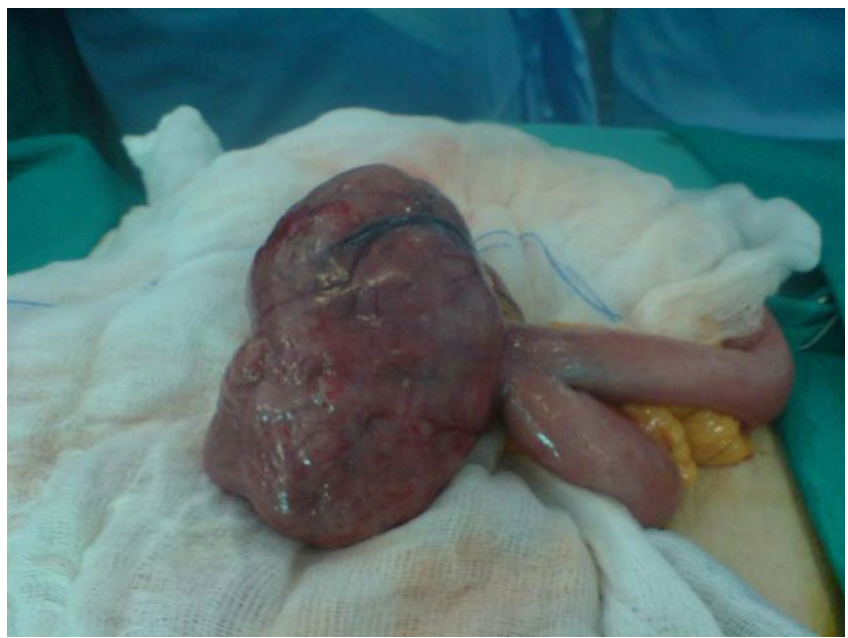

Figure 3

The well-circumscribed lesion of the jejunum before its removal. 
of varying differentiation [5]. GISTs account for only 1$3 \%$ of gastric tumours, $20 \%$ of small bowel tumours, and $1 \%$ or less of colorectal tumours. They have a predilection for adults older than 50 years, with the median ages varying around 60 years. Although these tumours usually develop in a sporadic fashion, familiar occurrence has also been reported [6].

Random genetic mutations are the apparent cause of GISTs. The majority of these tumours show identified mutations in cell-surface proteins called tyrosine kinase receptors [4]. Most GISTs show mutations in a gene that produces a growth factor receptor called KIT [6].

Primary GISTs may occur anywhere along the GI tract from the esophagus to the anus [7]. The most frequent site is the stomach (55\%), followed by the duodenum and small intestine (30\%), esophagus $(5 \%)$, rectum $(5 \%)$, colon $(2 \%)$, and rare other locations.

The most common presentation of GIST is acute or chronic gastrointestinal bleeding. They often present with nausea, vomiting, abdominal pain, metastatic diseases, and bowel obstruction. However, the symptoms depend on GIST location and size. Many tumours are found incidentally through medical imaging for other purposes or through surgery for other conditions. In our case the patient presented with anemia due to obscure bleea.

Many studies emphasize the CD117 and C5 expres. sion in GISTs [8]. A method of measurin of ho fast a GIST is growing is by the mitotic count sual examination of a set number of 'high power field (HPF) Jnder the microscope to count the number of tum crels caught in the process of mitosis or cell inion. The higher the mitotic count, the faster the turhor, growing. In this reported case, GIST was in rosed as a malignancy with moderate level of pho mo misn and mitotic activity while immunohistochen. and S-100 protei as posit, e.

The treatm nt of choic, is the complete resection of the tumour 5 si ged n's approach in an actual case depends on fartars su as. the exact anatomical site of the GIST, the cha cterist cs of the individual patient's particular situat. pecific location of the tumour relative to the blood oply of the involved organ, and the patient's condition. Patients with unresectable tumours or with metastatic disease are treated with Kit/PDGFRA tyrosine kinase inhibitors. Metastases may develop in the liver and the abdominal membranes. Patients with advanced GISTs which progress rapidly and result in organ destruction have poor prognosis.
In this reported case, the used diagnostic tools were abdominal US, upper gastrointestinal endoscopy, colonoscopy, abdominal CT, VCE and angiography. The first three investigations were unremarkable due the location of the tumour. The abdominal CT correctly imaged the location and the size of the tumour. It also excluded liver or peritoneal metastases and evaluated the ext nsion of the primary tumour. However, CT did not es al h the histological diagnosis of GIST. It is known that most GISTs have an exophytic growth, CT im ing is mol, useful than endoscopy and barium stu dies eval ate the extension and the size of the tumc ar [9]. It is o sensitive for the detection of metastatic le ons [10]. Lupescu et al [11] reported that using the $\mathrm{TT}$-an only suspect the diagnosis to GISTs. They efe at it is difficult to differentiate, using only CT ${ }^{*}$ aging, $t$ type of tumours from other soft-tissue one. Fi ${ }^{1 / y}$, Wu et al in a series of 100 small intestine ${ }^{-}$Ts refe , hat the sensitivity rate of abdominal CT vas $1 \%[12]$.

VCE reveled an ex Asive venous plexus giving the possible diagn an angiodysplasia of small intestine in our patient. Thi investigation did not establish the diagnosis of ${ }_{+1}$ mour $r$ ferring simply a benign abnormality. VCE did not lp the diagnosis in our case. In 29 centres of 10 urop ean countries, 5129 patients underwent VCE. 124 (. $10 \%$ ) of them had small bowel tumours [13]. The most common indication for VCE was obscure gastrointestinal bleeding (108 patients) while the main primary small bowel tumour type was GIST (32\%). These data suggested that VCE detected small-bowel tumours in a small proportion of patients underwent this examination. However, the early use of this diagnostic tool could shorten the diagnostic work-up and influenced the subsequent management of these patients [13]. Ziegler et al [14] reported that VCE identified small intestine lesions generally beyond the reach of push enteroscopy in $25 \%$ to $50 \%$ of patients and revealed additional diagnostic findings in $25 \%$ of patients compared with small bowel barium radiographic studies. The authors conclude that in some cases, the information provided by VCE in patients with obscure gastrointestinal bleeding can lead to changes in management that would improve their outcome. However, we did not use push enteroscopy and small intestine barium radiographic studies so we have not the ability to compare the results among these diagnostic tools.

In this reported case, angiography showed voluminous disorder with abnormal arterial structures and areas of stagnation of the contrast medium giving the diagnosis of a hypervascular malignant tumour without establishing the histological diagnosis of GIST. Despite there are not many studies regarding the use of VCE in diagnosis of GISTs, Fang et al [15] recently supported that VCE differentiate benign from malignant tumours, define their size, 
range and origin, despite the exophytic or endophytic growth of the tumours. However, they do not suggest routine use of VCE because of its risk and relative complications.

In conclusion, abdominal CT, VCE, and angiography were the diagnostic approaches we used for the GIST in our case report. It is known that the sensitivity of these investigations varied according to the location of the tumour. We found that CT was the most effective diagnostic method and we suggest routine use of this procedure in opposition to VCE and angiography which can give us some information but can also lead to changes in management that would not improve patient's outcome.

\section{Competing interests}

The authors declare that they have no competing interests.

\section{Authors' contributions}

DK and NS analyzed and interpreted the patient data. SA was the responsible doctor of the patient. SG was a major contributor in writing the manuscript. AK and IK were the radiologists who performed the angiography. All authors read and approved the final manuscript.

\section{Consent}

Written informed consent was obtained from the patent for publication of this case report and accompa. images. A copy of the written consent is avrilable review by the Editor-in-Chief of this journal

\section{References}

I. Nowain A, Bhakta H, Pais S, Kanel G, Ver S: Gasti ointestinal stromal tumors: clinical profile, path onesis. treatment strategies and prognosis. I Gastroe. Hepatol 2005, 20:818-824.

2. Eisenberg BL, Judson I: Surgery an III. ib in the management of GIST: emerging mproach $s$ to adjuvant and neoadjuvant therapy. Ann Surg Or 2004, 11:465-475.

3. DeMatteo RP, Lewis JI Ing Mudan SS, Woodruff JM, Brennan MF: Two hundred gas inesc...al stromal tumors: recurrence patterns nd prog, tis factors for survival. Ann Surg 2000, 23I:5I-5s.

4. Hirota $S$, Isoza. i K, ivama $Y$, Hashimoto K, Nishida $T$, Ishiguro $S$, Kawano K, Janada M, Kata A, Takeda M, Muhammad Tunio G, Matsuz- va $Y$, Kanakura/, Shinomura Y, Kitamura Y: Gain-of-function $n$ it ns f c-kit in human gastrointestinal stromal tumors. ance ' 98 , 279:577-580.

5. Ta MT, HB: Gastric stromal tumors. Reappraisal of hist renesis. Am J Surg Pathol 1983, 7:507-519.

$6 . \quad$ tirota S, Taniguchi M, Hashimoto K, Isozaki K, Nakamura h. anakura $\mathrm{Y}$, Tanaka T, Takabayashi $\mathrm{A}$, Matsuda $\mathrm{H}$, Kitamura $\mathrm{Y}$ : Far. al gastrointestinal stromal tumours with germline mutation of the KIT gene. Nat Genet 1998, 19:323-324.

7. Judson I: Gastrointestinal stromal tumours (GIST): biology and treatment. Ann Oncol 2002, 13(Suppl 4):287-289.

8. Hasegawa T, Matsuno Y, Shimoda T, Hirohashi S: Gastrointestinal stromal tumour: consistent CD I 17 immunostaining for diagnosis, and prognostic classification based on tumor size and MIB-I grade. Hum Pathol 2003, 33:669-676.

9. Sandrasegaran K, Rajesh A, Rushing DA, Rydberg J, Akisik FM, Henley JD: Gastrointestinal stromal tumors: CT and MRI findings. Eur Radiol 2005, I5: |407-|4|4.
10. Burkill G], Badran M, Al-Muderis O, Meirion Thomas J, Judson IR, Fisher C, Moskovic EC: Malignant gastrointestinal stromal tumor: distribution, imaging features, and pattern of metastatic spread. Radiology 2003, 226:527-532.

II. Lupescu IG, Grasu M, Boros M, Gheorghe C, lonescu M, Popescu I, Herlea V, Georgescu SA: Gastrointestinal stromal tumors: retrospective analysis of the computer-tomographic aspects. J Gastrointestin Liver Dis 2007, 16: I47-I45I.

12. Wu TJ, Lee LY, Yeh CN, Wu PY, Chao TC, Hwang TL, J , , IY, Chen MF: Surgical treatment and prognostic analysis $r$ gastrointestinal stromal tumors (GISTs) of the sina in sstir : before the era of imatinib mesylate. BMC Gastroe, 20,06 , 6:29-37.

13. Rondonotti E, Pennazio M, Toth E, Menchen 2 cioni Mi De Palma GD, Scotto F, De Looze D, Pachofsky Ta i I, Havelund T, Couto G, Trifan A, Kofokotsios A, Car nizzaro R, - Quadrado E, de Franchis R, European Capsule En sscopy Group Italian Club for Capsule Endoscopy (CICE); Iberian oup for Tapsule Endoscopy: Small-bowel neoplasms in $r$ tien. nder oing video capsule endoscopy: a multicenter $L$ ppea.. scudy. Endoscopy 2008, 40:488-495.

14. Ziegler KM, Flamm CR nnson N: reless capsule endoscopy in patients with o' scu. mall-intestinal bleeding. J Am Coll Radiol 2005, 2:818-820.

15. Fang SH, Dong J, ang SZ, JI, M: Angiographic findings of gastrointestin str nal tumor. World J Gastroenterol 2004, 10:2905-290,

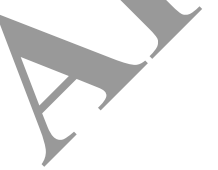

Publish with Bio Med Central and every scientist can read your work free of charge

"BioMed Central will be the most significant development for disseminating the results of biomedical research in our lifetime. "

Sir Paul Nurse, Cancer Research UK

Your research papers will be:

- available free of charge to the entire biomedical community

- peer reviewed and published immediately upon acceptance

- cited in PubMed and archived on PubMed Central

- yours - you keep the copyright

Submit your manuscript here:

http://www.biomedcentral.com/info/publishing_adv.asp
BioMedcentral 